Mots. Les langages du politique

$72 \mid 2003$

La ville, entre dire et faire

\title{
D'une identité politique à une identité communautaire
}

From political to communal identity

De una identidad política a una identidad comunitaria

\section{Britta Langhans}

\section{OpenEdition}

Journals

Édition électronique

URL : https://journals.openedition.org/mots/6433

DOI : $10.4000 /$ mots. 6433

ISSN : 1960-6001

Éditeur

ENS Éditions

Édition imprimée

Date de publication : 1 juillet 2003

Pagination : 135-156

ISBN : 2-84788-034-8

ISSN : 0243-6450

\section{Référence électronique}

Britta Langhans, «D'une identité politique à une identité communautaire », Mots. Les langages du politique [En ligne], 72 | 2003, mis en ligne le 29 avril 2008, consulté le 22 avril 2022. URL : http:// journals.openedition.org/mots/6433; DOI : https://doi.org/10.4000/mots.6433

\section{(c) ENS Éditions}




\section{D'une identité politique à une identité communautaire}

L'entretien que nous analysons a été effectué auprès de Marie, institutrice dans le village de La Bastide ${ }^{1}$, en décembre 1993, dans le cadre d'une enquête constituée de sept entretiens avec des habitants de ce village. Âgée de 50 ans au moment de l'interview, Marie habite son village natal, qu'elle a quitté seulement pour poursuivre ses études et le temps de ses premiers postes. À trente ans, elle est revenue à La Bastide. Cette étude nous permet d'illustrer deux idées.

D'une part, le fait que l'identité doit être appréhendée comme une construction discursive, instable, qui se modifie selon les rôles qu'une personne assume dans le dialogue et selon les objets qu'elle évoque ${ }^{2}$. La deuxième idée insiste sur le rôle de la narration ${ }^{3}$ : Marie justifie par l'histoire récente du collectif villageois un changement personnel profond.

- Université de Provence, «Analyse des fonctionnements langagiers», UMR CNRS 6057, Laboratoire Parole et Langage. britta.langhans@wanadoo.fr.

1. Les noms des personnes et des lieux ont été modifiés.

2. Pour étudier cette variation, nous avons réalisé une transcription minutieuse, capable de conserver toutes les traces du processus de l'activité discursive. Nous avons conservé les «ratés», les lapsus, les redémarrages, toutes les traces des difficultés posées soit par la recherche de l'expression adéquate (la résistance provient alors de la matérialité de la langue), soit par l'argumentation, soit par la dynamique de l'interaction...

3. Nous préférons parler de narration plutôt que de récit pour insister sur le caractère de production en train de se dérouler. La désignation récit peut en outre prêter à confusion: pour É. Benveniste, récit ou histoire renvoient à des caractéristiques formelles (l'emploi du passé simple et de la non-personne, $i l$, marque de la coupure entre l'énonciateur et le récit). Or, dans le cas présent, il n'y a aucune occurrence de passé simple et la narration est entièrement prise en charge par un je, notre corpus serait du discours pour Benveniste. L'étude s'écarte également de l'analyse structurale des années 70 qui s'intéressait au récit en tant que produit fini. Le caractère stable des unités dégagées était encore plus accentué lorsque les chercheurs analysaient des contes — récits fortement ritualisés — afin d'en dégager des structures globales. 
Elle se livre à une véritable construction identitaire. Ce qui nous intéresse dans l'analyse, ce n'est donc pas une reconstruction exacte de l'histoire mais la façon dont le sujet parlant s'inscrit dans sa reconstruction individuelle d'un vécu collectif.

L'observation de cette construction repose sur l'analyse du fonctionnement d'un certain nombre de marqueurs linguistiques à partir d'un nombre limité d'énoncés. Les marqueurs auxquels nous nous sommes intéressée se situent à deux niveaux. Premièrement, les marques de personne permettent de relever les postures énonciatives mises en place par la locutrice en fonction des sujets qu'elle évoque et des rôles identitaires qu'elle adopte. Deuxièmement, la pluralité des places occupées par Marie entrainant des prises de position parfois contradictoires, les connecteurs (mais, même...) constituent les traces linguistiques appréhendables de la production d'un sens dans le discours : concilier des points de vue déchirés, fluctuants en fonction de «l'autre» dont elle cherche à se démarquer.

\section{La trajectoire de Marie}

Afin de faciliter la compréhension du discours analysé, nous reconstruisons brièvement l'arrière-plan historique: dans le village de La Bastide - situé près d'Aix-en-Provence, à la limite des Bouches-du-Rhône et du Var - des changements importants de population sont intervenus à partir du début des années 70, marqués par une implantation massive d'habitants issus des villes proches, souvent des universitaires. Petit à petit, cette catégorie socioprofessionnelle a été relayée par l'arrivée d'une population plutôt ouvrière, issue également des villes, de Marseille surtout, et attirée par les prix de terrain intéressants.

Les intellectuels, d'une sensibilité de gauche, ont souhaité s'engager dans la politique municipale. Mais le maire en place — issu d'une famille où l'on était «maire de père en fils» — ayant refusé de faire liste commune avec eux, les nouveaux arrivants ont fini par présenter leur propre liste PS/PC. La liste des nouveaux arrivants a trouvé suffisamment d'alliés au sein des agriculteurs mécontents de la gestion de l'ancien maire pour emporter les élections municipales en 1977 et M. Dubreuil, leur candidat, devient maire de La Bastide pour deux mandats successifs (de 1977 à 1983 et de 1983 à 1989 ).

Pourtant, après quelques années, M. Dubreuil est désavoué par la population locale, mécontente, notamment, de la gestion du POS (plan d'occu- 
pation des sols). C'est cette période de l'histoire récente du village que Marie évoque pour expliquer comment son identité personnelle s'est modifiée. En effet, les évènements collectifs qui ont agité le village ont selon elle coupé la population en deux, séparant les villageois d'origine dont elle se rapproche et les nouveaux arrivants venus des villes dont elle pouvait paraitre proche par sa formation intellectuelle. Nous avons présenté ailleurs la façon dont Marie met en intrigue le scandale qui a déclenché le basculement de son identité politique et intellectuelle vers son identité locale (Langhans, 2000). Nous nous contentons d'en rappeler très brièvement quelques caractéristiques. La stratégie discursive de Marie consiste en une mise en scène d'un épisode qui lui permet d'affirmer qu'elle s'est métamorphosée de socialiste en Bastidoise et de justifier ce changement. Femme de gauche, Marie appréciait l'engagement politique des universitaires. Mais lorsque M. Dubreuil, leur tête de liste, irrité par les réticences du maire en place, a décidé de faire liste à part, Marie a changé de camp. Une phrase de Dubreuil sert de déclencheur «Nous dominerons La Bastide», et Marie souligne son effet de frontière. Elle passe alors d'une identité politique à une identité locale: «La Bastide [...] c'était moi». Pourtant, ses «identités» socialiste, rationnelle, ne disparaissent pas complètement. D'où une certaine instabilité de son positionnement qui traduit un éclatement de son sentiment d'appartenance collective.

\section{L'instabilité des positionnements}

L'identité est appréhendée ici comme une construction discursive où interfèrent d'une part les rôles ${ }^{4}$ assumés par la locutrice, d'autre part les objets dont elle parle; enfin, l'image qu'elle souhaite donner d'elle à l'enquêtrice.

\section{Instabilité des rôles identitaires de Marie}

Marie emploie une grande quantité de prédicats classifiants qui attribuent normalement des propriétés permanentes à un sujet ${ }^{5}$. Tantôt, elle se

4. Nous employons rôle dans un sens non technique. Les termes rôle, statut ou encore place seront utilisés sans tenir compte des débats interactionnistes.

5. Marie emploie également des termes traduisant des états affectifs ou émotionnels passagers (jamais satisfaite, effrayée, déçue). 
décrit par des termes qui la définissent par la référence au lieu (Bastidoise), tantôt elle se place d'un point de vue politique (socialiste, femme de gauche), ou d'un point de vue intellectuel (rationnelle). Or, comme la locutrice oscille constamment entre différentes classes, son positionnement discursif en devient extrêmement instable.

La Bastidoise. L'enracinement dans la terre par son ascendance lui confère clairement le statut de Bastidoise:

[1] je crois qu'ils [les enfants des nouveaux-arrivants] sont de La Bastide par rapport + les gens il faudrait peut-être le situer par rapport aux âges ça - - - par exemple moi je suis de La Bastide + maintenant je le suis par rapport à mes parents $[\ldots]$ à mes grands-parents même je dirais (p. 50-51) .

Ces origines rurales peuvent la rapprocher de personnes auxquelles elle s'opposerait en termes de convictions politiques:

[2] d'ailleurs je je dis toujours au maire en place tous ces problèmes que nous sommes pas du même bord + mais euh : + j'ai beaucoup plus d'affinités + avec une personne qui n'est pas de mon parti politique peut-être parce qu'il est de La Bastide - - - c'est quelque chose ça (p. 79).

Marie va jusqu'à s'identifier totalement au village lorsqu'elle cite la phrase malheureuse de M. Dubreuil, le candidat des nouveaux venus («nous dominerons La Bastide»):

[3] non mais + moi à ce moment-là + nous dominerons La Bastide + La Bastide c'était + c'était moi (p. 64).

L'intellectuelle. À d'autres moments, Marie se positionne comme une intellectuelle. Elle met en valeur l'importance des idées novatrices des universitaires arrivés des villes, et elle souligne elle-même son besoin de changement dans la gestion municipale des affaires culturelles :

[4] moi j'étais tout à fait + pour que + les gens qui arrivaient universitaires + euh + soient sur la liste de l'ancien maire soient là pour l'aider pour euh gérer le plan culturel + sur le plan culturel tout ça (p. 65).

6. Nous avons emprunté quelques symboles de transcription utilisés par le GARS (Claire Blanche-Benveniste 1990, p. 228): + pause courte; - - - pause longue;: allongement de voyelle; X syllabe incompréhensible ; que, Ø/ hésitation entre une écoute et rien /ces, ses/ alternances auditives ou orthographiques; les énoncés qui se chevauchent sont soulignés. Nous indiquons entre parenthèses la page du corpus dont est issu chaque extrait. 
Pour renforcer sa place d'intellectuelle, Marie attire l'attention de son interlocutrice explicitement sur son esprit rationnel:

[5] parce que je suis quand même rationnelle tu le sais bien $\mathrm{XX}+\mathrm{j}$ 'ai j'arrive à voir très bien les choses (p. 76).

La femme de gauche / la socialiste. Marie se définit à plusieurs reprises comme «femme de gauche», étant «socialiste par nature». Car son appartenance politique relève, selon elle, du même phénomène de filiation que son enracinement territorial.

[6] par contre alors moi là très ambigu encore + parce que moi qui suis quand même une femme de gauche enfin je crois + avec un père qui était socialiste et tout (p. 72).

[7] il y a un parti socialiste qui doit se réunir + à mon + mon insu parce que + c'est Dubreuil + c'est le clan Dubreuil et moi comme j'ai un peu rompu les les ponts quoique - - - pour moi c'est très ambigu parce que je suis socialiste par nature (p. 79).

Cette sensibilité de gauche provoque en elle un certain mécontentement par rapport à la mairie actuelle:

[8] et maintenant il y a une municipalité de droite + donc moi je suis jamais satisfaite (p. 69).

Cet échantillon illustre la pluralité des rôles de Marie. Aucun des autres enquêtés n'éprouve le besoin d'insister autant. Certes, chaque individu dispose de plusieurs places identitaires, mais on est rarement confronté à de telles variations d'images de soi, construites dans et par un seul discours.

\section{L'instabilité énonciative}

Cette instabilité peut dépendre de variations subtiles dans les objets dont parle Marie (c'est l'exemple des extraits [2] et [8] où s'opposent le lien à un individu de droite et le lien à un groupe de droite). Elle peut également renvoyer à des changements dans l'activité conversationnelle (l'évocation d'épisode «narratif» clé suscitant l'émotion).

Le conflit de la mairie résumé par Marie en tant qu'intellectuelle. En fonction des sujets abordés, Marie change ainsi de posture énonciative. Par exemple, elle résume dans un premier temps son conflit avec les nou- 
veaux arrivants en intellectuelle posée, produisant un discours didactique, assez critique vis-à-vis du comportement fermé des villageois; elle semble alors approuver l'engagement des universitaires.

[9] puis après euh : + des gens sont venus de l'extérieur + et se rendaient bien compte que ce maire-là n'évoluait pas en fonction + des idées nouvelles et qu'il y avait quand même beaucoup de choses à faire dans le village surtout sur le plan culturel donc quand les gens + quand d'autres personnes en plus des universitaires sont arrivées dans le village je crois qu'il y a eu un grand changement à partir de ce moment-là - - - et des gens qui cherchaient à s'intégrer au départ je crois + qui étaient pleins de bonne volonté comme ils n'arrivaient pas + à : + mettre les gens sous sous leur tutelle + les gens de La Bastide + ils n'arrivaient pas à les faire évoluer parce qu'on était terriblement ancré(s) on était + un peu: avare(s) on avait l'esprit euh + un peu + paysan je dirais + ils n'arrivaient pas à faire évoluer ces gens + et comme ils ne sont pas a- ils n'arrivaient pas à les faire évoluer je pense qu'ils avaient raison + ils ont essayé eux de prendre un peu le pouvoir + et de gérer les choses (p. 56-57).

Marie adopte d'abord un point de vue énonciatif extérieur («des gens sont venus de l'extérieur» et voulaient mettre «sous leur tutelle les gens de La Bastide»). Dans un commentaire critique vis-à-vis des villageois, elle justifie le comportement des intellectuels par la mentalité de la population rurale: «on était un peu avare, on avait l'esprit un peu paysan». L'utilisation du on introduit alors un changement de perspective: Marie semble s'inclure dans la communauté villageoise et s'opposer d'une manière floue à l'altérité construite par les gens / ils. À la fin de l'interview, Marie adopte à nouveau un point de vue distant pour décrire le comportement actuel des paysans vis-à-vis des nouveaux arrivants; elle emploie alors des génériques («quand on est paysan on est un peu avare») ${ }^{7}$ :

[10] les paysans ont vendu leur(s) terre(s) les paysans ont vendu leur(s) terre(s) parce que + ils sont quand même euh quand même + on est paysan on est toujours un peu un peu avare un peu + un peu pingre hein + un peu pingre oui c'est un peu ça et ils ont vendu les terres ça leur a rapporté énormément d'argent + parce qu'il faut bien dire que : - - les terres ne rapportent pl- + pas

7. Martina Drescher a élaboré une typologie des généralisations qui «fonctionnent [...] comme une espèce de joint entre différents épisodes thématiques» (1996, p. 140). Dans le corpus qu'elle analyse, les «paraphrases généralisantes constituent [...] un des moyens privilégiés pour segmenter le discours monologique», servant à «encadrer des unités narratives» $(1996$, p. 148). 
tellement hein + et les retraites sont très modestes alors ils ont vendu les terres mais maintenant ils regrettent et ils ne supportent pas que: + leur(s) terre(s) appartienne(nt) à des gens qui ne sont pas...(p. 84).

Le discours passe du on équivalent de nous [9] au on générique [10]. Paradoxalement, Marie - qui s'identifiait aux paysans en racontant l'histoire de la prise de la mairie, et qui affirme se définir désormais par son appartenance au terroir — va, sur le plan énonciatif, s'exclure du groupe des paysans, qu'elle décrit comme une catégorie, les paysans. $\mathrm{Au}$ lieu de s'attribuer une place définitive, Marie oscille entre intégration et distanciation.

L'échaudée. Lorsqu'elle procède à la mise en scène détaillée du scandale des élections municipales, Marie abandonne totalement son image rationnelle et adopte une attitude émotive («j'avais pas envie») :

[11] [...] mais quand j'ai vu après qu'ils menaient leur propre liste c'est à partir de ce moment-là où il $\mathrm{y}$ a eu rupture + parce que moi $\mathrm{j}$ 'ai euh tout de suite senti surtout après cette phrase + s'ils ne font pas liste commune du haut des Ormeaux nous dominerons La Bastide X ça j'aimerais bien que la mère Roux l'entende pardon + et bien euh - - - La Bastide moi j'avais pas envie parce que [...] La Bastide c'était La Bastide (p. 65-66).

Évoquer l'effroi ressenti en réaction à la phrase de Dubreuil suggère la gravité de la confiance et fait par contraste ressortir jusqu'à quel point Marie avait auparavant confiance dans les universitaires. À la différence des arguments politiques et culturels évoqués dans le premier paragraphe, il n'y a plus de place que pour les sentiments et la locutrice centre son discours autour du je:

[12] mais moi ce qui m'a vraiment effrayée cette phrase parce que au début je leur ai fait confiance + et je pensais qu'ils allaient [...] (p. 68).

Abandonnant son rôle d'intellectuelle distante, Marie décrit des émotions personnelles et elle pourra ainsi se fondre finalement dans la communauté villageoise.

La petite-fille d'agriculteurs. À d'autres moments, lorsqu'elle parle des traditions rurales et des rituels collectifs (le lavoir, la veillée, par exemple), Marie se pose en petite-fille d'agriculteurs. Ses souvenirs d'enfance imprégnés du mode de vie rural donnent une certaine légiti- 
mité à la place défendue par l'enquêtée, celle de la villageoise par ascendance.

[13] [...] parce que moi de tout ce qu'elle [la mère] raconte je me souviens par exemple du lavoir + je suis allée petite fille au lavoir + avec elle + je $:$ suis + j'ai assisté aux olives quand on nettoyait les olives le soir j'ai : j'ai assisté à ça pendant longtemps parce que jusqu'en $56+$ il y avait des olives $[\ldots]$ et puis + j'ai assisté + à la veillée proprement dite quand on allait chez la boulangère par exemple il y avait une fille qui avait 2 ou 3 ans de plus que moi + nous on était tranquillement euh: + en train de jouer pendant que les parents euh + buvaient le café (p. 14).

Cette description de l'enfance dans le village se transforme en revendication lorsque Marie s'oppose aux enfants des nouveaux arrivants, sa propre enfance lui servant d'argument:

[14] je les [les enfants] sens très différents + par exemple au moment de: + de la Noël là + euh on a parlé de la crèche euh + des traditions provençales + ils /me, nous/ regardent avec de grands yeux et même ceux qui sont de Marseille ce qui me parait curieux - - - un peu euh ne sont pas ne connaissent pas du tout les + la crèche + on fait la crèche mais ils ne connaissent pas du tout les traditions les Noëls provençaux par exemple - - - ils n'ont pas l'habitude de faire encore ça remonte plus loin + c'est que moi je suis vraiment du terroir j'ai: des parents + des grands-parents + des arrièregrands-parents qui sont nés dans ce village avec des traditions provençales très marquées (p. 48-49).

La population provençale, au lieu d'être unie par un facteur géographique commun, est au contraire inéluctablement divisée par la ruralité - perpétuant les traditions - qui constitue un trait identitaire prédominant. La ruralité se justifie par l'ascendance: «j'ai des parents + des grands-parents + des arrière-grands-parents qui sont nés dans ce village avec des traditions provençales très marquées », par conséquent, elle est «vraiment du terroir».

Ce rôle de Bastidoise se confirme à travers le sentiment des parents d'élèves issus de la ville («ils sentent aussi que je suis de La Bastide»):

[15] mais quand même quelquefois euh + je sens très bien que + ils sentent aussi que je suis + de La Bastide parce qu'ils se sentent pas toujours intégrés parce que nous on est pas toujours sympa avec eux + 'fin je dis nous euh + les gens les autochtones ne sont pas toujours sympathiques avec ces gens-là ils leur font sentir qu'ils ne sont pas de là + parce que contrairement + ils disent 
qu'ils sont fiers ils disent que: les les gens de La Bastide + mais en réalité ce sont des gens comme nous hein + faut pas non plus + d'autant plus qu'ils viennent pas de loin (p. 82).

En se voulant conciliante, Marie stigmatise encore davantage le groupe des nouveaux-arrivants (des gens): elle se positionne «en miroir», à l'intérieur du village, en s'intégrant de nouveau dans le nous: «en réalité ce sont des gens comme nous». Le contenu se trouve ainsi en contradiction avec le dispositif énonciatif. Une fois les nouveaux-arrivants mentionnés, elle s'y oppose en se renfermant à l'intérieur de son groupe d'appartenance. La démarcation énonciative (nous / ils) annule le fond de l'énoncé: ce sont des gens comme nous au lieu de nous sommes tous pareils. Le dispositif énonciatif oppose ce que le contenu semble unir; l'énoncé ne peut fonctionner qu'à partir d'une altérité posée comme différente.

La victime résignée des parents d'élèves. Son déchirement devient encore plus saillant lorsque l'enseignante parle des parents d'élèves. L'institutrice ne peut en fait se plaindre de l'engagement associatif de ces parents. Or la villageoise en elle se pose en victime: si tout se passe bien, c'est qu'elle a dû s'adapter.

[16] euh + l'association des parents d'élèves alors + l'association des parents d'élèves + est très + c'est-à-dire que moi + aussi j'ai dû un peu manigancer par rapport à la: l'évolution de la population + et j'ai dû m'adapter + parce que: bon hein + c'est pas quand même euh + il fal- il faut bien s'adapter (p. 77).

Si Marie trace une frontière énonciative (nous / ils) entre les nouveaux arrivants et les villageois, elle n'accepte pas pour autant de désigner les parents d'élèves par le terme étrangers. Elle l'attribue explicitement à on, puis à vous: sa mère a désigné ainsi les nouveaux arrivants, puis l'enquêtrice l'a imitée à plusieurs reprises dans l'entretien. La réfutation de cette désignation lexicale témoigne des scrupules de Marie à dire ouvertement la ségrégation qu'elle opère sans difficultés au niveau pronominal; car le choix pronominal est bien moins «visible». Le fait qu'elle n'a pas eu le choix lui confère le rôle de victime résignée:

[17] attention moi avec les étran- ce qu'on + ce que vous appelez les étrangers là + pour moi ça ne peut que bien se passer j'ai pas le choix de toute façon + ça je je vais : je suis au seuil au secret là + vous allez au fond de mes entrailles mais + euh: je veux dire que : + moi ça je veux dire /que, $\varnothing /$ ce sont des gens 
qui participent à la kermesse qui font tout + qui qui sont euh parce que je je je sais je sais pas - - - j'essaie de m'adapter (p. 82).

Cette résignation résulte apparemment d'une certaine antipathie que la villageoise éprouve à l'égard de ces parents issus des villes. Comme elle n'a rien de concret à leur reprocher, Marie n'exprime pas clairement les causes de son ressentiment. Elle justifie son mal à dire par l'appui sur l'affectif: «je suis au seuil au secret là; vous allez au fond de mes entrailles». Cette impossibilité de dire repose en partie sur les attentes vis-à-vis de l'enseignante, attentes liées aux images qui circulent dans l'interaction.

La relation enquêteur/enquêtée: co-construction du discours et des positionnements

L'image de l'enquêteur renforce la complexité des rôles et des perspectives adoptées.

Les explications destinées à l'interlocutrice. La présence de l'interlocutrice est à l'origine du discours de Marie: l'enquêtrice a sollicité cette évocation de l'histoire du village. Or, la présence d'une personne extérieure au conflit villageois provoque des sauts à l'intérieur de la construction, des oscillations constantes entre passé et présent, entre narration et commentaire.

[18] il y a quelques paysans qui se sont associés + qui se sont associés avec la liste Dubreuil + jusqu'à ce que Dubreuil reste en place + ensuite il y a eu un désaveu complet + puisque ces gens qui avaient fait + d'abord là c'est très important sur le plan sociologique parce que ces quelques jeunes qui : ont fait + qui avaient fait la liste avec Dubreuil là et qui étaient quand même pour lui et contre l'ancien maire (p. 66).

Dans le fil de la narration, Marie s'interrompt pour annoncer qu'un fait est important «sur le plan sociologique», puis elle donne des informations d'arrière-plan, au passé, mais qui ne font pas avancer l'intrigue en elle-même. Ces commentaires servent à assurer la compréhension, d'une part, à souligner ce qui semble intéressant, d'autre part.

Les images qui circulent. Marie et l'enquêtrice se connaissent depuis quelque temps, et elles partagent une sensibilité de gauche. Elles ont donc des attentes mutuelles liées aux images de soi et de l'autre. Ces images 
ont été construites en partie bien avant l'entretien, mais l'entretien incite les deux interlocutrices à les négocier. La réciprocité de ce processus a été mise en avant par Michel Pêcheux:

[...] ce qui fonctionne dans le processus discursif, c'est une série de formations imaginaires désignant la place que $\mathrm{A}$ et $\mathrm{B}$ s'attribuent chacun à soi et à l'autre, l'image qu'ils se font de leur propre place et de la place de l'autre (M. Pêcheux, 1969, Analyse automatique du discours, repris dans D. Maldidier, 1990, p. 118).

Lorsque certains de ses jugements dévient des principes liés aux rôles occupés habituellement, Marie doit renégocier sa place en produisant un certain nombre d'énoncés explicatifs. Regretter que ses élèves «ne soient plus ce qu'ils étaient avant» n'est pas conforme à la neutralité que l'on attend d'une éducatrice. Consciente d'aller à l'encontre de l'image de l'éducatrice, Marie formule alors son déchirement: "ça c'est terrible c'est très dangereux pour moi». Elle enchaine par un énoncé introduit par un mais restrictif, coupé de l'énonciateur (aucune marque formelle du je ne transparait), construit par une double négation: («mais ça ne veut pas dire qu'ils n'aient pas de valeurs ») :

[19] à travers les enfants d'aujourd'hui je ne peux pas - - - euh - - je regrette + en un sens je regrette qu'ils ne soient plus ce qu'ils étaient avant + parce qu'ils étaient ça c'est terrible c'est très dangereux pour moi c'est que : + ils ne ressemblent plus du tout à ce que j'attendais + et i- ils ils ont l'image est différente quoi + mais ça ça ne veut pas dire $[\ldots]$ ça ne veut pas dire qu'ils n'aient pas de valeurs (p. 58).

La préférence pour les enfants d'autrefois étant déontologiquement tout aussi injustifiable, Marie la motive par des critères scolaires objectifs tels que la discipline au travail. Ce n'est que par la suite qu'elle ajoute que ces élèves lui «ressemblaient étrangement».

[20] ils ressemblaient dans le comportement + ils savaient dire bonjour au revoir euh se tenir correctement euh pas euh - - - organiser même le travail aussi parce qu'ils étaient seuls à la maison et qu'ils n'avaient aucune aide et ce sont des enfants qui qui étaient encore des enfants de paysan(s) donc ils étaient un peu comme je ils avaient déjà changé parce que + le monde avait été changé certainement mais ils ressemblaient étran- + ils ressemblaient étrangement + ils ressemblaient étrangement à - - - à ce que j'étais + élève (p. 55).

Interventions actives. L'enquêtrice intervient à plusieurs reprises en posant des questions qui infléchissent la stratégie discursive de l'enquê- 
tée. Voici un exemple où elle met en doute l'unanimisme de la communauté. Marie doit bien avouer qu'au sein du groupe villageois existaient des clivages:

[21] Marie: [...] La Bastide moi j'avais pas envie parce que [...] La Bastide c'était La Bastide $[\ldots]$ ça appartenait + c'était + c'était très

Enq. : ça a été senti comme ça tu penses par tout le monde par des paysans que Marie: non ça alors + ça n'a pas été senti comme ça par tout le monde il y a quelques paysans qui se sont associés + qui se sont associés avec la liste Dubreuil [...] (p. 66).

L'enquêtée reprendra ensuite le fil de son discours. Mais il est indéniable que les questions de l'enquêtrice créent des ruptures supplémentaires dans la narration, révèlent des failles et amènent la locutrice à se justifier davantage.

\section{Les conflits de Marie: l'impossible à dire}

Le déchirement de la locutrice nait de l'impossibilité à dire ses sentiments intimes parce qu'ils sont en contradiction avec son image sociale, d'où un perpétuel processus de négociation et d'hésitation. À travers ses retournements, ses ruptures abruptes de positionnement, Marie s'efforce pourtant d'assurer une cohérence discursive. Nous pouvons relever les traces linguistiques qui renvoient aux opérations énonciatives par lesquelles Marie affaiblit ses assertions jusqu'à rendre conciliable l'inconciliable.

\section{Rectification de termes}

L'inadéquation de certaines appellations donne lieu à deux types de modulation: Marie affaiblit les termes par un peu, je crois... d'une part, ou elle procède à une réfutation explicite et à la recherche de mots plus justes. Le «transfert énonciatif» du on/nous au on générique (dans les exemples [9] et [10]) a été l'une des traces révélatrices de l'incertitude de Marie. Parallèlement, les énoncés témoignent d'un travail de non prise en charge des assertions. Toutefois, l'extériorité est à la fois lexicalement montrée par les termes pingre et avare et structuralement affaiblie par un peu. Ce terme, selon Oswald Ducrot, 
ne sert jamais à poser un jugement de quantité, mais seulement à délimiter quantitativement la portée d'un jugement. Alors que peu affirme une restriction, un peu restreint une affirmation (1972, p. 195).

L'emploi de un peu n'agit pas sur le contenu lexical, mais sur le degré de la prise en charge. Il est ainsi à rapprocher du commentaire énoncé en [6] où son auto-définition comme femme de gauche est aussitôt affaiblie par enfin je crois. Cette hésitation est la trace des négociations constantes dans le discours de Marie. Dans [15], l'extériorité des parents d'élèves récemment installés semblait renforcer le sentiment d'appartenance locale chez la locutrice. Or, dans la même séquence, Marie prend aussitôt ses distances par rapport aux autochtones: du on/nous elle passe à les gens / les autochtones, en s'en excluant: " nous on est pas toujours sympa avec eux + 'fin je dis nous euh + les gens les autochtones ne sont pas toujours sympathiques avec ces gens-là ils leur font sentir qu'ils ne sont pas de là ». À ce moment-là, Marie se distancie explicitement du groupe villageois: le commentaire méta-énonciatif 'fin je dis nous euh + les gens marque le refus de se mettre entièrement de leur côté. Le terme les gens sera rectifié à son tour par les autochtones, appuyant sur l'enracinement territorial.

Dans l'extrait [22], lorsqu'elle décrit la fête religieuse du village, Marie exprime son désir d'y participer. L'auto-désignation et même moi qui suis athée devait initialement renforcer l'importance de cette fête religieuse (introduction par même). Or, le terme athée est immédiatement rectifié:

[22] les vrais Bastidois si vous allez par exemple à cette neuvaine + vous /nous, le/ voyez le soir + et même moi qui suis athée + qui suis enfin qui suis athée + /je, qui/ ne suis pas athée mais pas du tout pratiquante [...] (p. 74).

De même, en suivant le fil du discours, nous voyons Marie affirmer son envie d'aller à la messe, puis rectifier:

[23] mais $+\mathrm{j}$ 'ai très + 'fin j'ai très envie + mais je veux vraiment on ne peut pas dire que ce soit une envie mais je veux dire que je me sentirais pas déplacée $[\ldots]$ (p. 75).

Dans tous ces exemples, lorsque Marie se rétracte, apparait le morphème enfin /'fin comme annonce linguistique d'une reformulation. 


\section{Ruptures syntaxiques}

Les amorces et les ruptures syntaxiques dans le discours de Marie témoignent du refus de mener une idée à son terme. Il s'agit de trouver un moyen de dire ce qui est inavouable.

[24] j'aurais presque envie + de participer à cette fête ça a tellement fait partie de: [...] si c'était pas que j'aie ce statut de de directrice d'école publique là + que je suis là depuis tout à l'heure 25 ans + et que on ne m'a jamais vu à la messe je peux pas y aller par rapport à euh + par rapport surtout aux gens de La Bastide + mais $+\mathrm{j}$ 'ai très + 'fin $\mathrm{j}$ ' ai très envie + mais je veux vraiment on ne peut pas dire que ce soit une envie mais je veux dire que je me sentirais pas déplacée $[\ldots]$ parce que ça ça fait partie d'une tradition ça + ça (p. 74-75).

Face à un sentiment inavouable compte tenu de l'image qu'elle défend, Marie s'interrompt sans cesse afin de trouver des formules adéquates. Comme déjà en [17], Marie s'interrompt dans l'extrait suivant pour indiquer qu'elle refuse d'assumer le terme étrangers:

[25] dans cette neuvaine là - - - les gens + qu'on appelle les étrangers puisqu'on les a appelés étrangers + ne participent + soit ils participent pas du tout

+ soit ils participent vraiment parce qu'ils sont très croyants (p. 74).

La rupture puisqu'on les a appelés étrangers permet d'atténuer le terme étrangers en indiquant qu'il doit être attribué au discours d'un «autre». Ainsi, Marie réussit à désigner un ensemble de personnes par étrangers sans prendre en charge le terme utilisé. Elle stigmatise un groupe et la rupture syntaxique lui permet de corriger immédiatement la prise de position liée à ce recours lexical.

\section{Les connecteurs argumentatifs : ceux qui permettent de dire}

L'abondance de connecteurs argumentatifs témoigne du souci d'assurer un lien formel entre des énoncés éclatés. Cette profusion renforce l'aspect contradictoire des prises de position de Marie. Certes, dans un discours narratif, «les organisateurs temporels [...] peuvent fort bien être combinés avec - voire remplacés par - des connecteurs argumentatifs » (Jean-Michel Adam, 1992, p. 71). De même, Jacques Bres a dégagé un changement de fonctionnalité que subit le connecteur parce que: le subordonnant, dans des récits oraux, devient 
[...] un outil métanarratif (au même titre que puis, alors, après, etc.) dont la fonction spécifique est d'expliciter la rupture temporaire de la mise en ascendance du temps raconté qui va se produire: une régression (1994, p. 169).

Mais ici ce sont avant tout les marques formelles d'un dispositif argumentatif qui structurent les bribes d'une narration biaisée par le refus d'assumer une position clairement déterminée. Les connecteurs argumentatifs permettent d'enchainer des points de vue contradictoires dans une structure cohérente pour dire l'indicible. Ces marques de l'argumentation sont repérables dans la structure de la phrase, comme le remarque O. Ducrot:

[...] la valeur argumentative d'un énoncé n'est pas seulement une conséquence des informations apportées par lui, mais la phrase peut comporter divers morphèmes, expressions ou tournures qui, en plus de leur contenu informatif, servent à donner une orientation argumentative à l'énoncé, à entrainer le destinataire dans telle ou telle direction (1980a, p. 15).

D'ailleurs : l'argumentation camouflée. Certains connecteurs, comme d'ailleurs et même, sont conformes à ce qu'on attend d'une argumentation. Dans [2], Marie fait allusion à sa bonne entente avec le maire de droite. Elle introduit son énoncé par d'ailleurs. Une question de l'enquêtrice l'oblige à expliquer son statut ambigu: non seulement la «socialiste par nature» a « un peu rompu les ponts » avec le clan socialiste, mais elle s'entend bien avec le maire de droite. Ce passage sert à étayer son développement basé sur l'enracinement local qui l'emporte sur les convictions politiques.

[26] Enq.: donc en gros + quand on parle associations: + et les partis politiques + il y a un Parti Socialiste qui continue à se réunir

Marie: il y a un parti socialiste qui doit se réunir + à mon + mon insu parce que + c'est Dubreuil + c'est le clan Dubreuil et moi comme j'ai un peu rompu les les ponts quoique - - - pour moi c'est très ambigu parce que je suis socialiste par nature - - et les choses ont + fait de telle façon que : + que je suis obligée + d'ailleurs je je dis toujours au maire en place tous ces problèmes que nous sommes pas du même bord + mais euh: $+\mathrm{j}$ 'ai beaucoup plus d'affinités + avec une personne qui n'est pas de mon parti politique peut-être parce qu'il est de La Bastide - - - c'est quelque chose ça (p. 79).

D'ailleurs fait apparaitre que les deux parties du raisonnement vont dans le même sens: 
L'élément $Q$ sur lequel porte d'ailleurs a toujours une valeur argumentative. C'est ce qui fait que d'ailleurs est impossible dans un contexte non argumentatif, lorsqu'on se contente d'inventorier un certain nombre de faits (O. Ducrot, 1980b, p. 197).

En même temps, d'ailleurs permet de camoufler l'argumentation:

Le locuteur prétend viser une conclusion $r$, il donne pour cette conclusion l'argument $P$ qui la justifie. Et, dans un second mouvement discursif, il ajoute un argument $Q$, allant dans le même sens que $P$. Dans la mesure où $P$ tout seul devait déjà conduire à $r, Q$ est ainsi présenté comme n'étant pas nécessaire pour l'argumentation. Le locuteur prétend ne pas utiliser $Q$ mais seulement l'évoquer (en d'autres termes, tout en présentant $Q$ comme un argument, il prétend ne pas argumenter à partir de $Q)$ (O. Ducrot, 1980b, p. 195).

Le processus argumentatif s'avère non avoué. Lorsque Marie décrit la procession de la fête religieuse, l'énoncé «elles la font toujours» est présenté comme une constatation. La marque d'ailleurs la transforme subrepticement en argument:

[27] et le jour de la fête du vœu par exemple + les femmes faisaient la procession + elles la font toujours d'ailleurs (p. 73).

Quand même: la rupture montrée. D'autres connecteurs argumentatifs moins ambigus s'ajoutent à la structure tissée par Marie. Ce sont les quand même qui dans ce discours signalent la difficulté qu'a Marie à se stabiliser dans une position. Malgré l'insistance sur l'égalité de tous les villageois (ce sont des gens comme nous), l'interviewée énumère des critères d'opposition à la fin de l'entretien. L'emploi de quand même marque alors la rupture d'avec ce qui a été dit auparavant:

[28] mais je crois que ce qui nous diffère quand même il y a un côté religieux + d'abord + qui est très ancré au fond de nous + qu'on ignore qu'on veut ignorer mais qui existe + et il y a une façon de vivre (p. 85).

Dans [6], son auto-définition politique révèle une rupture analogue, renforcée par l'emploi de par contre:

[6] par contre alors moi là très ambigu encore + parce que moi qui suis quand même une femme de gauche enfin je crois + avec un père qui était socialiste et tout (p. 72). 
Marie pose ainsi son appartenance à une catégorie comme inadéquate en même temps qu'elle se situe à l'intérieur de cette catégorie. Cet extrait s'insère dans un passage critiquant la mentalité de droite de la mairie. La socialiste Marie avouera par la suite «l'entente parfaite» avec le maire en place. Sa sensibilité politique va pourtant refaire surface en [29] lorsque l'enquêtée, adoptant de nouveau une perspective intellectuelle, dit apprécier les mouvements d'opposition de gauche au cœur du village:

[[29] et dans l'intérêt du village ce n'est pas très mauvais - - - qu'il y ait [...] des gens qui manifestent encore et qui ne disent pas amen à tout ce qui se fait parce que + c'est quand même une municipalité de droite (p. 76).

Dans l'extrait suivant, la contradiction marquée par quand même concerne moins les affirmations divergentes de la locutrice que le discours d'une altérité qui aurait voulu dissimuler les intérêts des «fidèles» de M. Dubreuil :

[30] deux ou trois qui avaient été fidèles et très favorisés + faut pas quand même + parce que nous l'histoire on la connait (p. 69).

Malgré sa déception vis-à-vis des intellectuels, Marie estime que leur engagement se justifiait par un besoin surtout culturel:

[31] il y avait quand même beaucoup de choses à faire dans le village surtout sur le plan culturel (p. 56).

À un autre moment, la contradiction montrée par quand même permet de souligner l'importance du désaveu des paysans:

[32] quelques jeunes qui : ont fait + qui avaient fait la liste avec Dubreuil là et qui étaient quand même pour lui et contre l'ancien maire (p. 66).

Le contraste de ces positionnements renforce la gravité de leur revirement final. Le connecteur quand même n'est plus la marque des contradictions de Marie mais la trace des transformations du collectif.

Les connecteurs mais et même: l'orientation des énoncés

Le mouvement de pensée dénoté par même est ainsi à peu près l'inverse de celui de mais. Mais l'un et l'autre ont en commun de faire allusion à la straté- 
gie du discours, à la façon dont les paroles prononcées orientent, ou risquent d'orienter, le débat des interlocuteurs (O. Ducrot, 1972, p. 130)

Leurs emplois permettent à la locutrice d'indiquer la visée des énoncés. Même, qui a un fonctionnement proche de d'ailleurs, est bien représenté dans le corpus. En [33], mentionner la présence des hommes, pourtant traditionnellement absents du rituel religieux en Provence, permet de renforcer l'importance de la fête religieuse:

[33] et même les hommes qui étaient Provençaux (p. 73).

Même indique à l'interlocutrice que cet énoncé va dans le même sens que ce qui précède. En [34], nous retrouvons cette orientation vers une même conclusion - l'importance de la fête:

[34] les gens des alentours venaient + même avec des cars (p. 74).

Marie va jusqu'à s'inscrire personnellement dans ce mouvement argumentatif; le rituel religieux attire une athée:

[35] et même moi qui suis athée (p. 74).

Ces énoncés portent la trace d'un effort visant à affermir des constatations :

Pour pouvoir dire $p$ et même $p$ ', il faut que $p$ et $p$ ' soient orientés vers une conclusion identique, et que $p^{\prime}$ y conduise mieux que $p$. Dans les deux cas, il y a bien une relation entre les informations apportées par les énoncés; mais cette relation, présentée comme purement factuelle dans le premier cas, passe nécessairement, dans le second, par les intentions argumentatives de celui qui parle (O. Ducrot, 1980a, p. 19).

En [36], Marie enchaine les deux connecteurs:

[36] le vœu c'était quelque chose de + tout à fait extraordinaire + mais même sur le plan religieux (p. 75).

Si même permet ici de renforcer le jugement de la locutrice, l'emploi de mais (qui a pourtant un fonctionnement opposé) sert à réfuter non pas ce qui précède mais plutôt la conclusion que l'enquêtrice pourrait tirer de l'engouement de Marie. L'énoncé introduit par mais répond à un non-dit: Marie, qui dit ne pas être pratiquante, a évoqué surtout la tradition locale qui confère à cette fête un statut extraordinaire. Pour- 
tant, elle l'est aussi d'un point de vue religieux. Mais réfute ce non-dit alors que même continue le mouvement argumentatif de ce qui vient d'être dit. On peut distinguer deux types d'emplois de mais dans les interventions de Marie:

— X mais $Y$ sert à réfuter un argument, à introduire un changement de position:

[37] moi j'étais tout à fait + pour que + les gens qui arrivaient universitaires + euh + soient sur la liste de l'ancien maire [...] pour gérer le plan culturel [...] mais quand j'ai vu après qu'ils menaient leur propre liste (p. 65).

[38] et soi-disant parce que en Provence les hommes [...] n'allaient pas à la messe mais ils respectaient tout ce qui était religieux (p. 73).

L'exemple [38] est orienté vers une conclusion analogue à celle de l'extrait [33]. Dans [39], mais introduit un changement de position en essayant de concilier la contradiction entre dire ne pas être pratiquante et exprimer son attachement au rituel religieux:

[39] ça c'est typiquement traditio- c'est lié à la religion en général + mais c'est c'est quelque chose que je vis profondément toutes les + toutes les années (p.75).

[40] les gens + qu'on appelle les étrangers [...] soit ils participent pas du tout + soit ils participent vraiment parce qu'ils sont très croyants + mais $:+$ les gens qui sont un peu indifférents euh + euh + sont complètement exclus de ça (p. 74).

La rectification : pas $X$ mais $Y$. Dans un certain nombre d'énoncés, la formule pas $X$ mais $Y$ témoigne des hésitations de la locutrice dues à l'éclatement de ses points de vue. Elle se rétracte en indiquant explicitement que des termes sont inadéquats. Dans les extraits suivants, athée et j'ai très envie sont ainsi rectifiés.

[22] [...] et même moi qui suis athée + qui suis enfin qui suis athée + /je, qui/ ne suis pas athée mais pas du tout pratiquante [...] (p. 74).

[23] mais + j'ai très + 'fin j'ai très envie + mais je veux vraiment on ne peut pas dire que ce soit une envie mais je veux dire que je me sentirais pas déplacée (p. 75).

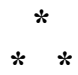


Marie se sert de l'histoire collective comme argument pour le changement personnel. Son histoire personnelle rejoint l'histoire collective par un premier biais; son discours se termine par l'évocation des villageois dissidents: à la fin de la narration, ils comprennent comme elle que le maire venu de l'extérieur était là pour dominer ${ }^{8}$ :

[41] mais au fur et à mesure des années quand ils ont vu + que le père Dubreuil était là $[\ldots]$ pour gérer le village [...] ils ont fait passer avant tout leur identité bastidoise + ils n'ont plus supporté + que le maire domine (p. 66).

Ainsi, le retournement de Marie est partagé par son groupe de référence principal. De cette façon, les évènements politiques ont donné lieu - toujours d'après notre locutrice - non pas seulement à un revirement personnel, mais à un revirement collectif. Marie réussit ainsi à mettre en place discursivement une communauté qui prend ses racines dans la lutte contre un «discours extérieur méprisant». Le clivage qu'elle construit repose en fait sur des évènements globaux: un traumatisme personnel, dû à une scène conflictuelle entre personnes. Cette scène devient alors symbolique de l'attitude des intellectuels «dominants». Dans son discours, Marie la transforme en évènement politique, puis local, enjeu d'identités qui cause en elle une rupture de convictions. Toutefois, Marie en face de son enquêtrice ne parvient pas à oublier ses autres communautés d'appartenance: l'Éducation nationale qui fait d'elle une institutrice laïque, les «intellectuels»... La question de son positionnement apparait dans toute sa complexité puisque Marie construit des catégories inconciliables. Les traces linguistiques témoignent du travail énonciatif visant à concilier des points de vue décousus, instables, variant en fonction de l'altérité à laquelle elle se confronte.

Ce sont les évènements politiques, dit-elle, qui l'ont placée dans cette position contradictoire. Or, les évènements auxquels elle réfère - ou plutôt, qu'elle construit dans et par son discours - apparaissent, de manière différente, dans tous les entretiens effectués et évidemment chez les amis de Dubreuil. L'évocation de cet épisode sert donc de point de fixation à son auto-analyse aussi bien qu'à toute la collectivité, qui y trouve un terrain d'entente paradoxal: un terrain commun pour s'opposer.

8. La façon de désigner les «dissidents» va de pair avec leur évolution: au début, Marie les appelle les ennemis du maire en place, puis elle reprend par ces paysans, ceux qui avaient fait liste commune, pour finalement arriver à ces quelques jeunes. 


\section{Références bibliographiques}

ADAM J. M., 1992, Les textes: types et prototypes, Paris, Nathan.

BLANCHE-BENVENISTE C., 1990, Le français parlé. Études grammaticales, Paris, Éditions du CNRS.

BRES J., 1994, La narrativité, Louvain-la-Neuve, Éditions Duculot.

DRESCHER M., 1996, «L'apport des généralisations à l'organisation du discours narratif», dans Laforest, 1996, p. 135-150.

DUCROT O., 1972 [1980], Dire et ne pas dire. Principes de sémantique linguistique, Paris, Hermann (coll. «Savoir»).

DUCROT O., 1980a, Les échelles argumentatives, Paris, Minuit.

DUCROT O. et al., 1980b, Les mots du discours, Paris, Minuit.

FOREST M. (dir.), 1996, Autour de la narration, CIRAL/Université Laval, Nuit Blanche Éditeur (coll. «Langue et pratiques discursives»).

LANGHANS B., 2000, «Narration et interview», Travaux n ${ }^{\circ} 15$, cercle linguistique d'Aix-en-Provence (CLAIX), Le récit, Publications de l'université de Provence, p. 29-47.

MALDIDIER D., 1990, L'inquiétude du discours, Paris, Éditions des cendres.

PÊCHEUX M., 1969, «Analyse automatique du discours», Paris, Dunod, 141 pages, extraits repris dans Maldidier, 1990, p. 97-132. 